II. Diskussion reaktiver Strategien 


\section{Impfen gegen COVID-19. \\ Die Stellungnahmen der Bioethikkommission beim Bundeskanzleramt als Beispiel für ethische Politikberatung}

Jürgen Wallner

\section{Einleitung}

Die SARS-CoV-2- bzw. COVID-19-Pandemie (im Folgenden immer nur kurz COVID-19-Pandemie) hat die Ethik vor eine breite Palette an Fragen gestellt (Kuczewski 2020; Simon/Zang 2020). Darunter solche der unmittelbaren klinischen Versorgung von an COVID-19 Erkrankten und die damit verbundenen Risiken einer Ressourcenknappheit (BÄK 2020; Bioethikkommission 2020e; DIVI 2020; Ehni et al. 2020; FASIM 2020; Hurst et al. 2020b); der Einschränkung oder Unterbindung von Kontaktrechten von Patient*innen in Krankenhäusern und Bewohner*innen in Langzeitpflegeeinrichtungen (Hurst et al. 2020a; Trad et al. 2020; Opolony 2021; Rogge et al. 2021); den Umgang mit moralischem Stress von Angehörigen der Gesundheits- und Sozialberufe (Knochel et al. 2020); der Herausforderung von mangelhafter Gesundheitskompetenz in der Bevölkerung (Czypionka et al. 2020; Rosner 2021); der Forschungsethik (Leopoldina 2020; Monrad 2020; Lane und Fauci 2021); oder von nationalen und internationalen Systemfragen der sozioökonomischen Einflussfaktoren auf Gesundheit (Deutscher Ethikrat 2020; Horton 2020a, 2020b; Lancet 2020). Insgesamt gewann durch die Pandemie die Public Health Ethik abrupt eine Relevanz, die ihr zuvor nur wenige Menschen zuerkannt haben (Childress et al. 2002; Hack 2020; Heilinger et al. 2020). Viele dieser ethischen Fragen wurden von Ethikberatungsgremien aufgegriffen: von Gremien wissenschaftlicher Akademien, Fachgesellschaften und Berufsvereinigungen sowie von solchen der ethischen Politikberatung. In Österreich wurde für die letztgenannte Aufgabe 2001 die Bioethikkommission beim Bundeskanzleramt (im Folgenden immer nur kurz Bioethikkommission oder Kommission) errichtet (Bioethikkommission 2020d). Sie berät den Bundeskanzler „in allen gesellschaftlichen, naturwissenschaftlichen und rechtlichen Fragen aus ethischer Sicht, die sich im Zusammenhang mit der Entwicklung der Wissenschaften auf dem Gebiet der Humanmedizin und -biologie 
ergeben.“ ( $\$ 2$ Abs. 1 BEK-V ${ }^{1}$ ) Damit vergleichbar beschäftigen sich in Deutschland der Deutsche Ethikrat und in der Schweiz die Nationale Ethikkommission im Bereich der Humanmedizin mit diesen Fragen und haben seit Beginn der COVID-19-Pandemie so wie die Bioethikkommission zahlreiche Stellungnahmen veröffentlicht. Der folgende Beitrag konzentriert sich auf jene Stellungnahmen, welche die Bioethikkommission zur Impfthematik erarbeitet hat.

\section{Vorarbeiten}

Die Bioethikkommission beschäftigte sich bislang in sieben Stellungnahmen mit ethischen Aspekten des Impfens, drei davon im Kontext der COVID-19-Pandemie. Im Folgenden werden diese Publikationen vorgestellt und durch weiterführende Literaturhinweise in die internationalen Fachdiskussionen eingebettet.

\subsection{Rahmenwerk der Impfethik}

Im Jahr 2014 wurde die Bioethikkommission vom Gesundheitsministerium um eine ethische Analyse des Themas Impfen ersucht. Anlass war die international geführte Diskussion um das vermehrte Auftreten von Masernfällen aufgrund eines Rückgangs der Inanspruchnahme von Masernschutzimpfungen (Schaks/Krahnert 2015; Sandhofer et al. 2017; Amhaouach/Kießling 2019; Rixen 2020). Daraus entwickelte sich die Stellungnahme „Impfen - ethische Aspekte“ vom Juni 2015 (Bioethikkommission 2015), in der die Kommission ein Rahmenwerk an Gesichtspunkten, Prinzipien und Kriterien für eine öffentliche Debatte darlegte, welches über die Masernimpfung hinausgeht. Der rote Faden, der sich durch diese und die folgenden Stellungnahmen durchzieht, ist die Zusammenschau von individual- und sozialethischer Perspektive (Kaelin 2016; Arora et al. 2018; Berlinger et al. 2020). Die klassische Medizin- und Bioethik ist durch die individualethische Auseinandersetzung mit der konkret betroffenen Person geprägt. Im Zusammenhang mit medizinischen Behandlungen, wie sie auch Impfungen darstellen, ist hierbei die Institution des Informed Consent maßgeblich. Demnach hat das betroffene Individuum das Recht,

1 Verordnung des Bundeskanzlers über die Einsetzung einer Bioethikkommission, BGBl. II 2001/226 i.d.F. BGBl. II 2012/335. 
in die Entscheidungsfindung eingebunden zu werden und eine Behandlung auch dann abzulehnen, wenn andere Menschen meinen, sie sei dringend empfohlen. Diese individualethische Herangehensweise, die sich insbesondere auf die ethischen Prinzipien des Respekts vor der Selbstbestimmung und des Nichtschadens berufen kann (Beauchamp/Childress 2019), gilt auch im Kontext von Impfungen. Zugleich sind zahlreiche Impfungen dadurch gekennzeichnet, dass sie nicht nur im Sinn der geimpften Person erfolgen, sondern ebenso zugunsten Dritter (Duttge 2019). Diese Berücksichtigung von Gruppen, der Bevölkerung und letztlich der Menschheit ist der für die Public Health Ethik typische sozialethische Ansatz (Dawson 2007; Colgrove 2019; Childress 2020). Er hat die öffentliche Gesundheit im Auge und zielt im Zusammenhang mit Impfprogrammen auf eine Gruppenimmunität ab, welche auch jene Personen vor einer Infektion bzw. Krankheit schützen soll, die nicht geimpft sind (Wiedermann-Schmidt 2016).

Individual- und sozialethische Perspektiven sind aufeinander angewiesen und können doch in Spannung zueinander stehen. Dies zeigt sich beispielsweise dann, wenn Individuen eine Impfung ablehnen und dadurch die Funktion gesellschaftlicher Bereiche (z. B. Gesundheits-, Bildungswesen) durch ein höheres Infektions- und damit Ausfallsrisiko der dort Tätigen gefährden. Hier würde die individualethisch begründete Entscheidung die sozialethisch begründeten Interessen unter Druck bringen. Umgekehrt wäre es, wenn ein Kollektiv Impfungen von Individuen allein mit der Überlegung erzwingt, dass dadurch Dritte einen Vorteil hätten. Hier würde die sozialethische Nutzenmaximierung individualethisch begründete Interessen negieren. Da somit weder individual-, noch sozialethische Gesichtspunkte eine pauschale, absolute Auflösung der Spannung erlauben, ist ein schonender Ausgleich berechtigter Interessen von Individuen und Kollektiven anzustreben. Diese Linie wird in der Stellungnahme der Bioethikkommission grundgelegt und differenziert entwickelt.

Ein erster Schritt besteht darin, dass der Ausgleich umso tragfähiger sein wird, je besser er sachlich begründet ist. Dies erfordert z. B. eine ausreichende Informationsgrundlage über die Wirkweisen von Infektionserregern hinsichtlich ihrer Transmission, Pathogenität oder Immunreaktionen der Infizierten und über die Nutzen-Risiko-Verhältnisse von konkreten Impfprogrammen im Allgemeinen und im Speziellen für bestimmte Zielgruppen (z. B. immunsupprimierte Personen). Ein zweiter Schritt fördert den Ausgleich dadurch, dass nach graduellen Lösungen gesucht wird, anstatt Absolutpositionen zu vertreten. Hierzu verweist die Stellungnahmen z. B. auf eine Kriteriologie (nachgewiesene Wirksamkeit, günstiges KostenRisiko-Verhältnis, akzeptables Kosten-Nutzen-Verhältnis, möglichst gerin- 
ge Restriktionen und faire, transparente Entscheidungsverfahren) und eine Eskalationsstrategie (rein private Entscheidung, Angebot mit Kostenübernahme, Empfehlung mit Kostenübernahme, Empfehlung mit positiven Anreizen, Pflicht mit negativen Anreizen). Viele dieser ethischen Überlegungen waren bereits zum Erarbeitungszeitpunkt der Stellungnahme in der österreichischen Rechtsordnung inkorporiert (Kopetzki 2017; Krasser 2020). So ist die Grundsatzabwägung zwischen individuellen und kollektiven Interessen in den Schranken und Eingriffsregeln der Europäischen Menschenrechtskonvention (EMRK) verankert. Die in der Judikatur dazu entwickelte Verhältnismäßigkeitsprüfung zielt auf den zuvor skizzierten Ausgleich ab. In den Worten der Bioethikkommission kann das Leitmotiv der Verhältnismäßigkeit von Impfregelungen wie folgt ausgedrückt werden:

Je harmloser der Eingriff für die einzelne Person ist, je ,gefährlicher die Krankheit für die Gesundheit der Bevölkerung ist und je größer der Nutzen einer Impfpflicht insgesamt ist, desto eher erscheint der Eingriff in die körperliche Integrität des Einzelnen gerechtfertigt. (Bioethikkommission 2015, 21)

Für die Bioethikkommission ist auf dieser Basis auch die schon damals geltende Eingriffsmöglichkeit einer Impfpflicht im Epidemiegesetz ${ }^{2}$ ausreichend begründet.

\subsection{Masern}

Im Mai 2019 konkretisierte die Bioethikkommission in einer Stellungnahme, dass sie die Verhältnismäßigkeit bei Masern als gewahrt ansieht und sich daher für eine allgemeine Impfpflicht in der Bevölkerung ausspricht (Bioethikkommission 2019).

\subsection{Pertussis, Pneumokokken, Influenza}

Vor dem Hintergrund dieser Grundsatzüberlegungen konnte die Bioethikkommission die im Zuge der COVID-19-Pandemie auftretenden Impffragen weiter bearbeiten. Dabei widmete sich die Kommission im Juni 2020 zunächst jenen Impfungen, die das Risiko eines schweren COVID-19- 
Krankheitsverlaufs senken sollen und für die es bereits zum damaligen Zeitpunkt zugelassene Impfstoffe gab: Pertussis (Keuchhusten), Pneumokokken-Infektionen und Influenza (Bioethikkommission 2020c). In Hinblick auf diese Impfungen sprach sich die Bioethikkommission für eine verstärkte Informationskampagne, finanzielle Unterstützung, einen generell niederschwelligen Zugang und eine Impfpflicht für bestimmte Berufsgruppen aus. Als wesentliches Argument führte die Kommission den Schutz jener Personen an, die sich selbst aufgrund ihres Gesundheitszustands oder Alters nicht impfen lassen können, obwohl sie es wollten. Zusätzlich wurde, jedenfalls für Pertussis, auf das Argument des Selbstschutzes der Geimpften verwiesen. In einer abschließenden Bemerkung sprach die Bioethikkommission in ihrer Stellungnahme ein weiteres ethisch relevantes Problem an: die Sicherstellung einer ausreichenden Impfstoffproduktion in Europa - ein Thema, das in der COVID-19-Pandemie noch hohe Bedeutung erhalten sollte.

\subsection{Influenza-Impfkampagne}

In Hinblick auf die Influenza-Schutzimpfung erinnerte die Bioethikkommission in einer weiteren Stellungnahme vom Oktober 2020 an die Bedeutung von fachlich fundierten Informationen über das Nutzen-RisikoVerhältnis (Bioethikkommission 2020a). Solche Informationen sollten, so die Kommission, durch die Kommunikation von Gesundheitsbehörden, Medien und Gesundheitsberufsverbänden vermittelt werden. Zusätzlich setzte sich die Bioethikkommission in dieser Stellungnahme mit der Frage auseinander, wie die Impfstoffe bei einer allfälligen Ressourcenknappheit allokiert werden sollten. Dabei verwies sie auf eine Empfehlung der Weltgesundheitsorganisation (WHO), wonach Angehörige von Gesundheitsberufen und Personen ab dem 60. Lebensjahr die höchste Priorität hätten, gefolgt von Personen mit chronischen Erkrankungen, Schwangeren und Kindern im Alter zwischen sechs und 59 Monaten. Um einer Mangelsituation in der Versorgung vorzubeugen (Williams/Dawson 2020), regte die Kommission eine vom Staat möglichst effizient gesteuerte Lieferkette von Zulassungsinhabern, Großhändlern und Apotheken an. Zusammenfassend erarbeitete die Bioethikkommission zu ethischen Aspekten des Impfens somit ein allgemeines Rahmenwerk (Juni 2015). Sie wandte dessen Methodik zunächst auf die Masern-Schutzimpfung an (Mai 2019). Schließlich erarbeitete die Kommission mit Hilfe des Rahmenwerks Analysen und Empfehlungen zu Impfungen gegen Pertussis, Pneumokokken und Influenza (Juni und Oktober 2020). 


\section{COVID-19-Impfung}

Seit Beginn der COVID-19-Pandemie wird sowohl an Medikamenten gegen die bereits ausgebrochene Erkrankung als auch an Impfstoffen zur Verhinderung einer Erkrankung geforscht. Die Bioethikkommission hat diese Entwicklung vor dem Hintergrund ihrer Vorarbeiten zur Impfethik in bislang drei Stellungnahmen begleitet.

\subsection{Ethische Fragen einer Impfung gegen COVID-19}

Im November 2020, also vor der Zulassung eines ersten COVID-19-Impfstoffs, veröffentlichte die Bioethikkommission ihre erste Stellungnahme hierzu (Bioethikkommission 2020b). Zum damaligen Zeitpunkt war noch unklar, ob, welchen bzw. wie viele, wann und wie es einen solchen Impfstoff geben wird. Diese unsichere Ausgangssituation gilt es auch in der ethischen Analyse wahrzunehmen. Deshalb ist die Stellungnahme der Bioethikkommission in vielen Punkten darauf angewiesen, mit Annahmen und Szenarien zu arbeiten, die durch den zum Zeitpunkt der Veröffentlichung bestmöglich verfügbaren Wissensstand belegt wurden (Nature Editors 2020; Schröder-Bäck et al. 2020).

\subsubsection{Allokation knapper Impfstoffe}

Unter der Annahme, dass es zu einem noch unbestimmten zukünftigen Zeitpunkt zumindest einen zugelassenen Impfstoff geben würde, widmete sich die Stellungnahme zunächst der Frage, welche Kriterien und Prozesse aus ethischer Sicht zu beachten sind, um die ohne Zweifel zunächst knappe Impfstoffmenge innerhalb Österreichs gerecht zu allokieren (Day et al. 2020; National Academies of Sciences 2020; Schmidt 2020; Berlinger et al. 2021; Hughes et al. 2021; Shachar 2021). Die Empfehlungen für eine Allokation basieren auf einer Zusammenschau von fachlichen (pharmakologischen, medizinischen, epidemiologischen) und normativen (rechtlichen, ethischen) Gesichtspunkten. Die Berücksichtigung fachlicher Aspekte ist für die ethische Urteilsbildung nötig, weil eine gerechte Allokation unter anderem davon abhängt, wie die individuelle Nutzen-Risiko-Abwägung für die impfbereite Person aussieht und welche Effekte die Impfung für Dritte hätte. Hier zeigt sich die schon zuvor im Rahmenwerk angesprochene Doppel-Perspektive von individual- und sozialethischer Relevanz der 
Impffragen. Je nachdem, wie ein konkreter Impfstoff wirkt (z. B. nur einen schweren Krankheitsverlauf verhindert, das Risiko eine Infektion der geimpften Person senkt oder eine Übertragung des Virus unterbindet), können unterschiedliche Personengruppen in unterschiedlicher Weise von Impffragen betroffen sein und sind diese folglich auch unterschiedlich bei Allokationsfragen zu berücksichtigen. Die Stellungnahme weist darauf hin, dass diese Aspekte zum damaligen Zeitpunkt noch nicht ausreichend mit Inhalten befüllt werden konnten, da noch kein Impfstoff eine Zulassung beantragt oder erhalten hatte. Zugleich zeigt die Stellungnahme, dass auch unabhängig davon eine fundierte und vorausschauende Systematik entwickelt werden kann, die dann im Anlassfall rechtzeitig für akute Entscheidungen heranzuziehen ist.

Die normativen Aspekte einer Allokation von COVID-19-Impfstoffen werden von der Bioethikkommission vor dem Hintergrund des geltenden Gesundheits- und Gleichbehandlungsrechts erörtert. Die hierbei einschlägigen Prinzipien von Autonomie, Nichtschaden, Wohltun und Verantwortung führen zu folgender sachlich begründeter Differenzierung und Priorisierung von Personengruppen bei der Impfstoffallokation:

1. Vulnerable Personen: Die höchste Priorität sollten jene Menschen haben, deren Risiko, an einer COVID-19-Erkrankung schwer zu Schaden zu kommen, am größten ist. Ziel wäre es, deren individuelle Krankheitslast, aber auch eine durch ihre Behandlungsbedürftigkeit entstehende Belastung des Gesundheitssystems, möglichst niedrig zu halten. Die Vulnerabilität kann durch hohes Alter, spezifische Vorerkrankungen, den aktuellen Gesundheitszustand oder die psychosoziale Lebenssituation bedingt sein.

2. Gesundheits- und Pflegepersonal: Die zweithöchste Priorität sollten Angehörige der Gesundheits- und Sozialberufe erhalten. Dies begründet sich zum einen damit, dass diese Personen durch ihre Berufe ein erhöhtes Infektionsrisiko haben (also selbst erkranken können); zum anderen dadurch, dass die Sicherstellung ihrer Versorgungsleistung für alle anderen Menschen wichtig ist und die Infektion von Gesundheitsund Pflegepersonal selbst bei einem individuell symptomlosen oder milden Verlauf zu dessen Ausfall in der Versorgung führt.

3. Multiplikatoren: Die dritthöchste Priorität sollten jene Gruppen haben, die aufgrund eines hochfrequenten Kontakts zu anderen Menschen selbst und für diese anderen ein erhöhtes Infektionsrisiko haben. Dazu zählen zum Beispiel Berufe, die mit Kindern und deren Eltern regelmäßig zusammenkommen (Kindergärten, Schulen) und Dienstleistungsberufe, die mit vielen, aber nicht näher bekannten Personen zu tun 
haben (etwa Angestellte in Supermärkten). Begründen lässt sich diese Priorisierung neben dem individuellen Schutz der geimpften Person mit dem Ziel, das Infektionsgeschehen durch die Immunisierung von potenziellen Multiplikatoren einzudämmen und damit auch Dritte zu schützen.

4. Beschäftigte in kritischer Infrastruktur: Die vierthöchste Priorität hätten jene Gruppen, die für die Aufrechterhaltung des Gemeinwesens besonders relevant sind. Die Bioethikkommission verweist darauf, dass deren Identifikation bzw. Eingrenzung umstritten sein kann, aber wohl jedenfalls die Bereiche Sicherheit (z. B. Gesundheitsämter, Polizei, Feuerwehr) und Versorgung (z. B. Energie, Wasser, Müll) umfassen.

Ein ethisch relevanter Aspekt der Allokation knapper COVID-19-Impfstoffe wird in der Stellungnahme der Bioethikkommission nicht behandelt: die Frage der globalen Allokation. Sie wird in den internationalen Fachpublikationen seit Auftreten von COVID-19 intensiv diskutiert (Bollyky/Bown 2020; Emanuel et al. 2020; Usher 2020; Emanuel et al. 2021). Denn die Art, wie COVID-19-Impfstoffe global allokiert werden, drückt Vorstellungen von Gerechtigkeit aus und stellt eine wahrscheinlich entscheidende Weiche in der Effektivität der Pandemiebekämpfung dar.

\subsubsection{Freiwilligkeit und ibre Grenzen}

Ein zweites Thema, dem sich die Stellungnahme der Bioethikkommission vom November 2020 widmet, betrifft jenes Szenario, in dem COVID-19Impfstoff zur Verfügung steht, aber die Nachfrage der Menschen geringer ist als das Angebot (Rosenbaum 2021). Die Bioethikkommission nähert sich dem international seit langer Zeit sehr intensiv debattierten Thema einer Impfpflicht durch die Differenzierung zwischen einer allgemeinen Impfpflicht und einem Impfnachweis als Voraussetzung für die Ausübung bestimmter Tätigkeiten (z. B. in einem Beruf) (Heissenberger 2016; Wünsch-Brandner/Grandl-Eder 2019). In Hinblick auf eine COVID-19Impfung als Berufsausübungserfordernis verweist die Bioethikkommission auf ihre bereits weiter oben dargestellten Vorarbeiten. Besondere Aufmerksamkeit widmet sie den Schutz- und Organisationspflichten der Träger von Gesundheits- und Sozialeinrichtungen (Grimm 2016; Berlinger et al. 2021). Demnach kann die Impfentscheidung in einer arbeitsteiligen Organisation, die einer über die Individualverantwortung hinausgehenden institutionellen Gesamtverantwortung unterliegt, nicht bloß vom Individuum abhängig gemacht werden. Zugleich ist eine Organisation darauf ver- 
pflichtet, einen Interessensausgleich zwischen den berechtigten Präferenzen ihrer Angestellten und anderer Stakeholder (v. a. ihren Klient*innen) zu versuchen. Die Bioethikkommission anerkennt, dass dieser Versuch (z. B. durch Einsatz von nicht-geimpftem Personal in Klient*innen-fernen Bereichen) an Grenzen stoßen wird. Zwar setzt sich die Stellungnahme nicht mit einschlägigen arbeitsrechtlichen Konfliktmechanismen (z. B. Kündigung) auseinander, sie verweist aber auf die schon seit langem existierende Möglichkeit des Epidemiegesetzes, eine Impfung als Berufsausübungserfordernis für das Gesundheitspersonal einzufordern und durch eine entsprechende rechtliche Regelung zu normieren. In Hinblick auf eine allgemeine Impfpflicht für die Bevölkerung bezieht sich die Bioethikkommission auf ihr Rahmenwerk, insbesondere auf die Methodik und den Prüfmaßstab der Verhältnismäßigkeit. In Anbetracht der noch unklaren fachlichen Aspekte der COVID-19-Impfstoffe sieht die Bioethikkommission die Verhältnismäßigkeit für eine allgemeine Impfpflicht zum Zeitpunkt der Stellungnahme als noch nicht beurteilbar und eine Pflicht daher als nicht gerechtfertigt an. Stattdessen sprach sich die Bioethikkommission für eine umfassende und transparente Information über alle Schritte der Zulassung, Allokation und Anwendung künftiger COVID-19-Impfstoffe aus, um so der Bevölkerung möglichst autonome Entscheidungen über die individuelle Impfbereitschaft zu ermöglichen.

\subsubsection{Einschränkungen von Grundrechten}

Abschließend beschäftigt sich die Bioethikkommission in dieser Stellungnahme bereits mit der weiterführenden Frage, wie sich die COVID-19Impfung auf die Ausübung von Grundrechten in der Pandemie auswirken könnte. Seit Beginn der Pandemie wurden in Österreich und international in die Ausübung einiger Grundrechte einschränkend eingegriffen (z. B. Freizügigkeit, Privatleben, Erwerbstätigkeit). Mit Einführung einer Impfung und dem dadurch beeinflussten epidemiologischen Geschehen würden sich wesentliche Gesichtspunkte der Erforderlichkeit und damit Verhältnismäßigkeit dieser Einschränkungen verändern. Schon in dieser Stellungnahme vom November 2020 wies die Bioethikkommission darauf hin, dass die Rechtfertigung für Grundrechtsbeschränkungen somit unter Hinweis auf einen positiven Impfstatus für die betroffenen Individuen wegfallen müsste. Zugleich sprach die Bioethikkommission das sozialethische Problem an, dass mit dieser individualethisch solide begründeten, vollen Wiederherstellung der Freiheitsrechte eine gesellschaftliche „Demoralisierung" einhergehen könnte, der es gegenzusteuern gelte z. B. durch 
Aufrechterhaltung von allgemeinen Schutzmaßnahmen wie Hygiene- und Schutzmaskenpflichten.

\subsection{Rechtliche und ethische Fragen im Zusammenhang mit geimpften und genesenen Personen in der COVID-19-Pandemie}

Im April 2021 knüpfte die Bioethikkommission an jenes Thema an, welches sich in ihrer Stellungnahme vom November 2020 bereits abgezeichnet hatte: Welche Aspekte sind zu berücksichtigen, wenn es um den staatlichen Umgang mit geimpften oder genesenen Personen in der COVID-19-Pandemie geht? (Bioethikkommission 2021b) Anlass war eine Anfrage des Gesundheitsministeriums, welches mit der Vorbereitung eines Immunitätsnachweises (Grüner Pass) beschäftigt war, der in Österreich und der Europäischen Union den Status von COVID-19-Impfung und -Genesung dokumentieren sollte (Brown et al. 2020; de Miguel Beriain/Rueda 2020; Kofler/Baylis 2020; Persad/Emanuel 2020; Phelan 2020; Ravitsky/Weinstock 2020; Council of Europe 2021; Hall/Studdert 2021). Ausgangspunkt der Überlegungen der Bioethikkommission waren wiederum die fachlichen (pharmakologischen, medizinischen, epidemiologischen) Grundlagen, die im April 2021 schon deutlich klarer waren als zum Zeitpunkt der vorangegangenen Stellungnahme. Mittlerweile wurden weltweit mehrere Impfstoffe eingesetzt, die allesamt eine sehr hohe Wirksamkeit gegen Hospitalisierung, schwere Verläufe und Tod bei COVID-19 zeigten. Zusätzlich zum Selbstschutz der Geimpften verringerten die Impfstoffe das Risiko einer Übertragung des Virus (Transmissionsschutz), hatten also einen Nutzen für (auch nicht-geimpfte) Dritte. Die Risiken, die mit einer COVID-19-Impfung einhergehen können (z. B. starke Impfreaktionen oder Nebenwirkungen) wurden in der Stellungnahme nicht weiter beleuchtet, da sie sich ausschließlich der Frage widmete, was eine erfolgte Impfung für die Betroffenen in Hinblick auf ihre Grundrechte bedeuten kann. Da sich für von COVID-19 Genesene und negativ Getestete analoge Fragen stellen, wurden diese Personengruppen in der Stellungnahme ebenfalls berücksichtigt.

Normativer Bezugspunkt der Überlegungen ist der grundrechtliche Rahmen, wie er für Österreich insbesondere durch die EMRK bestimmt wird. Einschränkungen der darin enthaltenen Individualrechte wurden und werden in einer Epidemie- oder Pandemiesituation in der Regel mit dem Schutz der Gesundheitsversorgung und dem Schutz Dritter gerechtfertigt. Die Schwierigkeit, dass diese Einschränkungen gegenüber jedem einzeln davon betroffenen Menschen zu begründen sind, dies aber 
zugleich an praktische Grenzen stößt (z. B. der Schwierigkeit, das individuelle Risiko hinreichend zuverlässig zu bestimmen oder der Schwierigkeit, die Einhaltung individuell abgestimmter Verhaltensregeln zu überprüfen), muss laut Bioethikkommission in der Verhältnismäßigkeitsprüfung berücksichtigt werden. Im Ergebnis führt diese Methodik dazu, dass Grundrechtseinschränkungen zurückgenommen werden müssen, soweit nicht zwingende Gründe praktischer Art, der gesellschaftlichen Destabilisierung (,Demoralisierung ${ }^{6}$ ) oder eines gesteigerten Schutzbedürfnisses von speziellen Gruppen ( $\mathrm{z}$. B. hochvulnerablen Personen) dagegensprechen. Die Politik steht vor diesem Hintergrund vor der Herausforderung, das grundsätzlich als richtig Erkannte klug umzusetzen. Dazu gehört insbesondere, Effekte zu vermeiden, die letztlich zu einer Erosion des für die Aufrechterhaltung der (Grund-)Rechtsordnung unabdingbaren Rechtsethos nötig sind. Deshalb sieht die Bioethikkommission die politische Verantwortung bei der Ausgestaltung eines Immunitätsnachweises nicht nur dahingehend, eine formal korrekte grundrechtliche Umsetzung sicherzustellen, sondern zusätzlich dabei auf jene Politikinstrumente zurückzugreifen, die jenes Rechtsethos unterstützen, das für die Umsetzung vorausgesetzt werden muss. Dazu zählt laut Stellungnahme insbesondere die Sensibilisierung für Solidaritätserfordernisse innerhalb der Gesellschaft und die Stärkung der Gesundheitskompetenz in der Bevölkerung, um mit (Infektions-)Risiken differenziert umgehen zu können (Abstiens et al. 2021). Aufgrund der fachlichen Erkenntnisse sprechen somit starke Argumente dafür, dass die grundrechtlichen Einschränkungen für geimpfte und genesene Menschen so rasch und so weit wie möglich zurückzunehmen sind, bei gleichzeitiger umsichtiger politischer Gestaltung des Umgangs mit dem weiterhin bestehenden epidemiologischen Risiko und mit den Auswirkungen der Regulierung auf das Rechtsethos. Ein Baustein, den die Bioethikkommission in diesem Zusammenhang anspricht, ist die immunologische Barrierefreiheit, d. h. die möglichst niederschwellige Möglichkeit der Testung und Gleichstellung von Getesteten mit Geimpften bzw. Genesenen, solange eine Impfung nicht allgemein verfügbar ist.

Der Eindruck, dass mit der Wiederherstellung der Grundfreiheiten für Geimpfte, Genesene und Getestete jene diskriminiert würden, die nicht zu einer dieser Gruppen zählen, entkräftet die Bioethikkommission. Soweit die zuvor genannten Aspekte der immunologischen Barrierefreiheit erfüllt sind, werden Menschen, die nicht zu den Geimpften, Genesenen oder Getesteten zählen, nicht unsachlich anders behandelt (d. h. diskriminiert), sondern allenfalls sachlich begründet differenziert (in Hinblick auf gesundheitliche Risiken für Dritte). Im Gegenteil würde vor diesem Hintergrund die Aufrechterhaltung der Freiheitsbeschränkungen für Ge- 
impfte, Genesene und Getestete deren Diskriminierung darstellen, und zwar im Vergleich zu jenem Normalfall der vollen Freiheitsrechte, die nur sachlich begründet und verhältnismäßig eingeschränkt werden dürfen. Dem Vorwurf, dass dadurch eine indirekte Impfpflicht für alle Menschen begründet würde, begegnet die Bioethikkommission mit einer Differenzierung: Einerseits anerkennt sie, dass durch das beschriebene Vorgehen die Anreize zur Impfung steigen. Soweit damit Menschen, die sich nicht impfen oder testen lassen wollen, aber nicht von der Befriedigung fundamentaler Grundbedürfnisse ausgeschlossen werden, wie sie typischerweise mit einem Kontrahierungszwang belegt sind (z. B. öffentlicher Verkehr, Lebensmittelversorgung, Apotheken), trifft der Begriff ,Impfpflicht ${ }^{6}$ die Situation weder formal noch inhaltlich korrekt. Abschließend weist die Bioethikkommission darauf hin, dass die Instrumente zum Nachweis einer COVID-19-Impfung, -Genesung oder -Testung nur so lange eingesetzt werden sollten, als dies durch die epidemiologische Lage erforderlich ist.

\subsection{Impfung gegen COVID-19 als Berufserfordernis für die Gruppe der Pflege- und Gesundheitsberufe}

In ihrer Stellungnahme vom Mai 2021 griff die Bioethikkommission eine Frage heraus, die sie schon in vorangegangenen Publikationen analysiert hatte: jene nach dem Impfstatus von Angehörigen der Gesundheits- und Sozialberufe (Bioethikkommission 2021a). Die Stellungnahme stellt in gewisser Weise eine Bekräftigung bisheriger Aussagen vor dem Hintergrund weiter fortschreitender Erkenntnisse zum Pandemie-Verlauf und den Wirkweisen sowie Nutzen-Risiko-Profilen der zugelassenen COVID-19-Impfstoffe dar. Die Bioethikkommission erinnert daran, dass dem Gesundheitspersonal eine sehr hohe Priorität zukommen sollte, wenn es um die Allokation von Impfstoffen geht. Dies soll den Betroffenen ermöglichen, möglichst effizient ein Impfangebot wahrnehmen zu können. Sodann verweist die Kommission auf ihre Beurteilung, wonach eine COVID-19-Impfung zumindest für die Dauer der Pandemie als Voraussetzung für die Berufsausübung eingefordert werden sollte. Im Unterschied zu einer allgemeinen Impfpflicht, welche die Bioethikkommission weiterhin als nicht erforderlich ansieht, spricht sie sich für eine solche Berufspflicht aus. Anlass für diese Bekräftigung waren anhaltende Impflücken beim Personal von Gesundheits- und Pflegeeinrichtungen, die zu einem weiterhin bestehenden Infektionsrisiko und Einschränkungen der Freiheit für (geimpfte) Klient*innen führten. Vor diesem Hintergrund verwies die Bioethikkommission nochmals auf die ohnedies im Epidemiegesetz bestehen- 
de Möglichkeit, eine Impfung als Voraussetzung für die Ausübung eines Gesundheitsberufs zu normieren. Unter Berücksichtigung der Verhältnismäßigkeit wird die politische Verantwortung, eine solche Normierung in Erwägung zu ziehen, umso schwerwiegender, je klarer die Geeignetheit (Wirksamkeit der Impfung), Notwendigkeit (nachdem gelindere Mittel des Anbietens, Empfehlens ausgeschöpft sind) und Adäquanz (in Hinblick auf das Nutzen-Risiko-Profil des konkreten Impfstoffs) wird. Dass diese Verhältnismäßigkeit auch innerhalb der Gesundheitsberufe nochmals zu einer sachlichen Differenzierung führen kann (z. B. durch Abstufungen nach Gefährdungsklassen), ist für die Bioethikkommission ein zusätzliches Argument, diese Schritte in Erwägung zu ziehen.

\section{Fazit: Ethische Politikberatung am Beispiel der COVID-19-Impfung}

Die COVID-19-Pandemie stellt alle Akteure vor zahlreiche, weitgefächerte ethische Herausforderungen. Soweit es sich bei den Akteuren um politische Verantwortungsträger handelt, können diese auf Gremien und Instrumente der ethischen Politikberatung zurückgreifen, wie sie sich vor allem seit Beginn der 2000er-Jahre international im Zuge der Entwicklung der biomedizinischen Forschung (Life Sciences) etabliert haben. In Österreich wird diese ethische Politikberatung auf Ebene der Bundesregierung durch die Bioethikkommission wahrgenommen. Die Bioethikkommission hat sich in den letzten Jahren mehrfach und intensiv mit ethischen Aspekten des Impfens auseinandergesetzt. Im Kontext der COVID-19-Pandemie kamen dabei klassische impfethische Topoi wie das Verhältnis von Selbstbestimmung und Verantwortung gegenüber Dritten ebenso vor wie zusätzliche Aspekte, etwa allokationsethische Fragestellungen. Die Bioethikkommission griff diese verschiedenen Themen auf und versuchte, sie innerhalb eines nachvollziehbaren ethischen Beurteilungsrahmens zu analysieren. Dieser Beurteilungsrahmen umfasst den Abgleich von deskriptiven, prognostischen, evaluativen und normativen Aspekten und Argumenten, die zusammen zu ethischen Urteilen führen (Wallner 2018). Kein Aspekt für sich allein kann ein ethisches Urteil ausreichend begründen. Deshalb ist die ethische Urteilsbildung nicht nur eine vielschichtige, sondern auch aufwendige Angelegenheit. Wer hier ,Abkürzungen' sucht und allein auf Basis des Faktischen, denkbarer Szenarien, Präferenzen oder Normen ein ethisches Urteil bilden möchte, wird zu kurz greifen und schlimmstenfalls in die Irre gehen (z. B. durch Moralisieren gegenteilige Effekte erzielen). Die Bioethikkommission versucht, die verschiedenen Aspekte der ethischen Urteilsbildung durch ihre multiprofessionelle und multidisziplinäre 
Zusammensetzung abzudecken. Die Struktur und Argumentation ihrer Stellungnahmen sind der Nachweis, inwieweit ihr das gelingt.

Inwieweit die Bioethikkommission dieser Aufgabe gerecht wird, zeigt sich daher in den Hauptteilen ihrer Stellungnahmen: in den Analysen, Argumentationen und Abwägungen. Sie liefern jene Substanz, die man gemeinhin mit ethischer Beratung verbindet: eine Unterstützung bei der eigenen (autonomen) Urteilsbildung. Die Rezipienten der Stellungnahmen, zumal wenn sie Entscheidungsträger sind, fokussieren freilich meist auf die Empfehlungen der Bioethikkommission. Dies ist insofern verständlich, als sich Entscheidungsträger unter Beratung auch das Einbringen von Expertise erwarten, die ihnen einige Optionen nahelegt (zurät, empfiehlt) und von anderen abrät. Diese Form von Expertise (Fachexpertise) ist gerade im Kontext der COVID-19-Pandemie von vielen Seiten kritisch beleuchtet worden (Kissler 2020; Lübbe 2020). Aus ethischer Perspektive erfolgt diese Kritik insoweit zurecht, als die Grenzen zwischen Expertise und Entscheidungsautorität verschwimmen, d. h. Fragen der Legitimität und Verantwortung unbeantwortet bleiben. Für die Ethikberatung kommt hin$\mathrm{zu}$, dass ihre Expertise von manchen Menschen darin gesehen wird, dass ethische Expertise zwingende normative Gründe für eine Handlungsoption darlegen sollte. Wo es solche zwingenden Gründe gibt, ist Ethikberatung aber tendenziell nicht nötig (da die Situation ohnehin klar genug ist) oder sie droht zum Feigenblatt verschobener Verantwortung zu werden.

In Hinblick auf das Thema der COVID-19-Impfung ist die Lage allerdings ohnehin alles andere als eine durch und durch zwingender Gründe. Die „Bürden des Urteilens" sind fachlich und ethisch hoch (Rawls 2003, $\$ 11.4$ ). Wie sich in der Analyse der Stellungnahmen der Bioethikkommission gezeigt hat, durchzieht sie wie ein roter Faden der Versuch, verschiedene gut begründete Gesichtspunkte zu einem schonenden Ausgleich zu bringen: individual- und sozialethische Aspekte oder Zielvorstellungen und Normen, die Freiheiten und Sicherheiten betreffen. Dabei gibt es viele Differenzierungen, Abstufungen und Abwägungen. Ethische Politikberatung kann in diesem Zusammenhang ein Instrumentarium entwickeln, das Kriterien und Prozesse beschreibt, wie diese grundsätzlich gleichbegründeten Gesichtspunkte miteinander in Beziehung zu setzen sind. Konkretisiert man dies, etwa am Beispiel der COVID-19-Impfung, zeichnen sich einige Handlungsoptionen ab, die ethisch besser begründet werden können als andere - mitunter auch so gut, dass der Eindruck entstehen kann, es sprechen eindeutig zwingende Argumente für oder gegen eine Option. Doch selbst in diesem Fall sollte ethische Politikberatung stets eine selbstkritische Zurückhaltung üben: Wenn sie (von Politikverantwortlichen oder Medien) gefragt wird, was sie denn „empfehle“ oder als 
„ethisch" ansehe, müsste die Antwort eigentlich stets in folgende Richtung gehen: „Wir erachten folgende Gesichtspunkte für maßgeblich, um sich ein Urteil zu bilden ... Das haben auch wir selbst getan und können damit folgende Empfehlungen argumentieren ... Es ist aber nicht ausgeschlossen, dass andere Menschen unter Beachtung derselben Kriterien zu anderen Schlussfolgerungen kommen." - Mitunter zeigt sich dies auch innerhalb der Bioethikkommission, wenn unterschiedliche Schlussfolgerungen publiziert werden (die hier erwähnten Stellungnahmen sind alle einhellig verabschiedet worden). - Liest man sich die Stellungnahmen zum Thema (COVID-19-)Impfung durch, so gewinnt man hoffentlich den Eindruck, dass die Bioethikkommission diese Spannung von Erwägung und Empfehlung nicht aus dem Auge verloren hat.

\section{Literatur}

Abstiens, K./Czypionka, T./Gangl, K./Grosch, K./König, T./Spitzer, F./Walter, A. (2021): Zehn Gebote der Verhaltenswissenschaften in der Pandemiebekämpfung, in: http://irihs.ihs.ac.at/5702/ [09.06.2021].

Amhaouach, L./Kießling, A.: Die Steigerung der Masernimpfraten von Kindern Möglichkeiten und Grenzen des Rechts, in: Medizinrecht 37/11 (2019) 853-861. DOI: $10.1007 / \mathrm{s} 00350-019-5366-9$.

Arora, KS./Morris, J.Jacobs, AJ.: Refusal of Vaccination: A Test to Balance Societal and Individual Interests. Journal of Clinical Ethics 29/3 (2018) 206-216.

BÄK: Orientierungshilfe der Bundesärztekammer zur Allokation medizinischer Ressourcen am Beispiel der SARS-CoV-2-Pandemie im Falle eines Kapazitätsmangels (2020), in: https://www.bundesaerztekammer.de/fileadmin/user_uploa d/downloads/pdf-Ordner/Stellungnahmen/BAEK_Allokationspapier_05052020. pdf [09.06.2021].

Beauchamp, T.L./ Childress, J.F.: Principles of Biomedical Ethics. New York, NY: Oxford University Press ${ }^{8} 2019$.

Berlinger, N./Wynia, M./Powell, T./Milliken, A./Khatri, P./Marouf, F./Ray, K./Crane, J.: Ethical Challenges in the Middle Tier of Covid-19 Vaccine Allocation: Guidance for Organizational Decision-Making (2021), in: https://www.thehastingscenter.org/ethical-challenges-in-the-middletier-of-covid-19-vaccine-allocation/ [09.06.2021].

Berlinger, N./Wynia, MK./Powell, T./Hester, DM./Milliken, A./Fabi, RE./Cohn, F./ Guidry-Grimes, LK./Watson, JC./Bruce, L./Chuang, EJ./Oei, G./Abbott, J./Jenks, NP.: Ethical Framework for Health Care Institutions \& Guidelines for Institutional Ethics Services Responding to the Coronavirus Pandemic. Managing Uncertainty, Safeguarding Communities, Guiding Practice (2020), in: https:// www.thehastingscenter.org/ethicalframeworkcovid19/ [09.06.2021]. 
Bioethikkommission: Impfen - ethische Aspekte (2015), in: https://www.bundeska nzleramt.gv.at/dam/jcr:1b8fb237-fc34-450c-840f-58918c73154a/Impfen\%20und \%20ethische\%20Aspekte.pdf [07.06.2021].

Bioethikkommission: Stellungnahme der Bioethikkommission zum Impfen (2019), in: https://www.bundeskanzleramt.gv.at/themen/bioethikkommission/pressem itteilungen-bioethik/stellungnahme-der-bioethikkommission-zum-impfen.html [07.06.2021].

Bioethikkommission: Empfehlung „Versorgung der Bevölkerung mit dem Influenza-Impfstoff in der Saison 2020/21“ (2020a), in: https://www.bundeskanzleramt. gv.at/themen/bioethikkommission/pressemitteilungen-bioethik/empfehlung-ver sorgung-der-bevoelkerung-mit-dem-influenza-impfstoff-in-der-saison-2020_21.ht $\mathrm{ml}[07.06 .2021]$.

Bioethikkommission: Ethische Fragen einer Impfung gegen COVID-19 (2020b), in: https://www.bundeskanzleramt.gv.at/dam/jcr:d92558b8-c664-46a5-af86-d4f6994 a9ec8/201127_StN_COVID_Impfstoff.pdf [30.11.2020].

Bioethikkommission: Impfen gegen Erkrankungen, für die es zugelassene Impfstoffe gibt, in Zeiten der COVID-19-Pandemie (2020c), in: https://www.bundeskanz leramt.gv.at/dam/jcr:97856399-b564-47ba-8d34-8a621b778d33/200622_Stellung nahme_COVID_Impfen_A4_BF.pdf [07.06.2021].

Bioethikkommission: Tätigkeitsbericht der Bioethikkommission: Oktober 2019Oktober 2020 (2020d), in: https://www.bundeskanzleramt.gv.at/dam/jcr:991ff3e c-eba8-4d9a-98f2-fc673b4463c9/taetigkeitsbericht_bioethikkommission_2019-20 20.pdf [09.06.2021].

Bioethikkommission: Zum Umgang mit knappen Ressourcen in der Gesundheitsversorgung im Kontext der Covid-19-Pandemie (2020e), in: https://www.bundes kanzleramt.gv.at/dam/jcr:772d37b0-3db9-4c8b-b4fe-e6dca7b1b8d3/200402_Covi d_Bioethik.pdf [08.11.2020].

Bioethikkommission: Impfung gegen COVID-19 als Berufsausübungserfordernis für die Gruppe der Pflege- und Gesundheitsberufe (2021a), in: https:/www.bun deskanzleramt.gv.at/dam/jcr:b777e891-d79e-4a9a-9cf0-943995263e06/Stellungna hme_Impfung_Gesundheitsberufe_052021.pdf [07.06.2021].

Bioethikkommission: Rechtliche und ethische Fragen in Zusammenhang mit geimpften und genesenen Personen in der COVID-19-Pandemie (2021b), in: https://www.bundeskanzleramt.gv.at/dam/jcr:ba1f33aa-9a50-4e4f-a7d0-2e58e ef34c89/Stellungnahme_COVID_April_2021.pdf [07.06.2021].

Bollyky, TJ./Bown, CP.: The Tragedy of Vaccine Nationalism, in: Foreign Affairs 99/5 (2020) 96-100.

Brown, RCH./Savulescu, J./Williams, B./Wilkinson, D.: Passport to Freedom? Immunity Passports for COVID-19, in: Journal of Medical Ethics 46/10 (2020) 652659. DOI: 10.1136/medethics-2020-106365.

Childress, J.F.: John Stuart Mill's Legacy for Public Health Ethics. In: Ders.: Public Bioethics. New York, NY: Oxford University Press 2020, 289-317. 
Childress, J.F./Faden, RR./Gaare, RD./Gostin, LO./Kahn, J./Bonnie, RJ./Kass, NE./ Mastroianni, AC./Moreno, JD./Nieburg, P.: Public Health Ethics: Mapping the Terrain, in: Journal of Law, Medicine \& Ethics 30/2 (2002) 170-178. DOI: 10.1111/j.1748 - 720X.2002.tb00384.x.

Colgrove, J.: Immunization and Ethics: Beneficence, Coercion, Public Health, and the State, in: Mastroianni, AC./Kahn, JP./Kass, NE.: Oxford Handbook of Public Health Ethics. New York, NY: Oxford University Press 2019, 435-447.

Council of Europe (2021): Protection of Human Rights and the "Vaccine Pass", in: Information Documents, SG/Inf(2021)11, in: https://rm.coe.int/0900001680a1fac4 [06.06.2021].

Czypionka, T./Reiss, M./Röhrling, G.: Identifikation von Faktoren, die ex-ante zur Resilienz einer Volkswirtschaft gegen gesundheitlich ausgelöste wirtschaftliche Krisen beitragen - Teilaspekt Gesundheitssystem (2020), in: https://irihs.ihs.ac.at /id/eprint/5621 [09.06.2021].

Dawson, A.: Vaccination Ethics, in: Ashcroft, RE./Dawson, A./Draper, H./McMillan, JR.: Principles of Health Care Ethics. Chichester, Wiley: ${ }^{2} 2007,617-622$.

Day, RT./Guidry, BS./Drolet, BC./Clayton, EW.: From Ventilators to Vaccines: Reframing the Ethics of Resource Allocation, in: American Journal of Bioethics 20/7 (2020), W15-W16. DOI: 10.1080/15265161.2020.1782530.

de Miguel Beriain, I./Rueda, J.: Immunity Passports, Fundamental Rights and Public Health Hazards: A Reply to Brown et al, in: Journal of Medical Ethics 46/10 (2020) 660-661. DOI: 10.1136/medethics-2020-106814.

Deutscher Ethikrat: Solidarität und Verantwortung in der Corona-Krise: Ad-hocEmpfehlung (2020), in: https:/www.ethikrat.org/mitteilungen/2020/solidaritaet -und-verantwortung-in-der-corona-krise/ [09.06.2021].

DIVI: Entscheidungen über die Zuteilung von Ressourcen in der Notfall- und der Intensivmedizin im Kontext der COVID-19-Pandemie Version 2 (2020), in: https:/www.awmf.org/leitlinien/detail/11/040-013.html [09.06.2021].

Duttge, G.: Patientenautonomie auch zur Vorbeugung gegen Infektionskrankheiten? Wieviel individuelle (Ir-)Rationalität kann sich ein gesamtgesellschaftlicher Gesundheitsschutz leisten?, in: Bernat, E./Grabenwarter, C./Kneihs, B.S. et al.: Festschrift Christian Kopetzki. Wien: Manz 2019, 129-140.

Ehni, H-J./Wiesing, U./ Ranisch, R.: Entscheidungen unter akutem Ressourcenmangel: Europäische Triage-Empfehlungen in der COVID-19-Pandemie, in: Zeitschrift für medizinische Ethik 66/4 (2020) 475-488. DOI: 10.14623/zfme.2020.4.475 - 488.

Emanuel, EJ./Fabre, C./Halliday, D./Leland, R.J./Buchanan, A./Tan, K-C./Chang, S.Y.: How Many Vaccine Doses Can Nations Ethically Hoard? (2021), in: https:// www.foreignaffairs.com/articles/world/2021-03-09/how-many-vaccine-doses-can -nations-ethically-hoard [09.06.2021].

Emanuel, EJ./Persad, G./Kern, A./Buchanan, A./Fabre, C./Halliday, D./Heath, J./ Herzog, L./Leland, RJ./Lemango, ET./Luna, F./McCoy, MS./Norheim, OF./Ottersen, T./Schaefer, GO./,Tan, K-C./Wellman, CH./Wolff, J./Richardson, HS.: An Ethical Framework for Global Vaccine Allocation, in: Science 369/6509 (2020) 1309-1312. DOI: 10.1126/science.abe2803. 
FASIM: Allokationsethische Orientierungshilfe für den Einsatz knapper intensivmedizinischer Ressourcen (2020), in: https:/www.intensivmedizin.at/sites/defau lt/files/konsensus_fasim_allokation_intensiv_finale_fassung_text_11.11.2020.pdf [09.06.2021].

Grimm, M.: Verpflichtende Impfung von Mitarbeitern im Gesundheitsbereich?, in: Aigner, G./Grimm, M./Kletečka-Pulker, M./Wiedermann-Schmidt, U.: Schutzimpfungen - Rechtliche, ethische und medizinische Aspekte. Wien: Verlag Österreich 2016, 85-108.

Hack, C.: Ethikberatung und Covid-19: Brauchen wir mehr Ethik, mehr als Ethik oder mehr Ethiker?, in: Zeitschrift für Praktische Philosophie 7/2 (2020) 279-306. DOI: 10.22613/zfpp/7.2.12.

Hall, MA./Studdert, DM.: "Vaccine Passport" Certification - Policy and Ethical Considerations, in: New England Journal of Medicine (2021). DOI: 10.1056/ NEJMp2104289.

Heilinger, J-C./Thompson, A./Wild, V.: Public Health Ethics and Covid-19: The Ethical Dimensions of Public Health Decision-Making during a Pandemic (2020), in: https:/www.public-health-covid19.de/images/2020/Ergebnisse/Policy_Brief_Pandemic_Ethics_GeneralF.pdf [09.06.2021].

Heissenberger, W.: Impfen in Österreich - Überlegungen zur Impfpflicht und Darstellungen de lege lata, in: Aigner, G./Grimm, M./Kletečka-Pulker, M./Wiedermann-Schmidt, U.: Schutzimpfungen - Rechtliche, ethische und medizinische Aspekte. Wien: Verlag Österreich 2016, 53-84.

Horton, R.: Offline: A Global Health Crisis? No, Something Far Worse, in: Lancet 395/10234 (2020a) 1410. DOI: 10.1016/S0140-6736(20)31017-5.

Horton, R.: Offline: COVID-19 Is Not a Pandemic, in: Lancet 396/10255 (2020b) 874. DOI: 10.1016/S0140-6736(20)32000-6.

Hughes, MT./Kahn, J./Kachalia, A.: Who Goes First? Government Leaders and Prioritization of SARS-CoV-2 Vaccines, in: New England Journal of Medicine 384/5 (2021) e15(1-2). DOI: 10.1056/NEJMpv2036128.

Hurst. SA./Belser, EM./Burton-Jeangros, C./Mahon, P./Hummel, C./Monteverde, S./Krones, T./Dagron, S./Bensimon, C./Schaffert, B./Trechsel, S./Chiapperino, L./Kloetzer, L./Zittoun, T./Jox, RJ./Fischer, M./Dalle Ave, AL./Kirchschläger, PG./Moon, S.: (2020a). Continued Confinement of Those Most Vulnerable to COVID-19, in: Kennedy Institute of Ethics Journal 30/3-4 (2020a) 401-118. DOI: $10.1353 /$ ken.2020.0021.

Hurst, SA./Fumeaux, T./Heise, A./Schaffert, B./Perrier, A./Rütsche, B./Krones, T./ Gruberski, T./Ackermann, S./Salathé, M./Scheidegger, D.: Covid-19-Pandemie: Triage von intensivmedizinischen Behandlungen bei Ressourcenknappheit. Hinweise zur Umsetzung Kapitel 9.3 der SAMW-Richtlinien (2020b), in: https:// www.samw.ch/dam/jcr:14cb0e53-eedb-4089-96dd-b49a1b388994/richtlinien_v3 -1_samw_triage_intensivmedizinische_massnahmen_ressourcenknappheit_2020 1217.pdf [15.01.2021]. 
Kaelin, L.: Impfen - Zwischen Autonomie und Gemeinwohl, in: Aigner, G./ Grimm, M./Kletečka-Pulker, M./Wiedermann-Schmidt, U.: Schutzimpfungen Rechtliche, ethische und medizinische Aspekte. Wien: Verlag Österreich (2016) 223-230.

Kissler, A.: Ein Ethikrat ist nicht die alles entscheidende moralische Instanz (2020), in: https:/www.nzz.ch/meinung/ein-ethikrat-ist-nicht-die-alles-entscheidende-m oralische-instanz-ld.1593272 [09.06.2021].

Knochel, K./Kühlmeyer, K./Richstein, K-H./Rogge, A.: Psychische Belastungen von Gesundheitspersonal im Umgang mit moralischen Konflikten: Eine Handreichung für klinische Ethikberater*innen (2020), in: https://aem-online.de/file admin/user_upload/HandreichungPsychischeBelastungen2020-03-31.pdf [09.06.2021].

Kofler, N./Baylis, FE.: Ten Reasons Why Immunity Passports Are a Bad Idea, in: Nature 581 (2020) 379-381. DOI: 10.1038/d41586-020-01451-0.

Kopetzki, C.: Impfpflicht und Verfassung, in: Recht der Medizin 24/2 (2017) 45.

Krasser, A.: Zur grundrechtlichen Zulässigkeit einer Impfpflicht, in: Recht der Medizin 27/4 (2020) 136-142.

Kuczewski, MG.: Measure Twice and Cut Once: The Value of Health Care Ethicists in the Pandemic (2020), in: https://www.thehastingscenter.org/measure-twiceand-cut-once-the-value-of-health-care-ethicists-in-the-pandemic/ [09.06.2021].

Lancet: COVID-19: Remaking the Social Contract, in: Lancet 395/10234 (2020) 1401. DOI: 10.1016/S0140-6736(20)30983-1.

Lane, HC./Fauci, AS.: Research in the Context of a Pandemic, in: New England Journal of Medicine 384/8 (2021) 755-757. DOI: 10.1056/NEJMe2024638.

Leopoldina: Coronavirus-Pandemie: Medizinische Versorgung und patientennahe Forschung in einem adaptiven Gesundheitssystem (4. Ad-hoc Stellungnahme) (2020), in: https://www.leopoldina.org/uploads/tx_leopublication/2020_05_27_S tellungnahme_Corona_Gesundheitssystem.pdf [09.06.2021].

Lübbe, W.: Orientierung in der Corona-Krise? Nicht mit Doppelbotschaften, in: Medizinrecht 38/6 (2020) 434-439. DOI: 10.1007/s00350-020-5557-4.

Monrad, JT.: Ethical Considerations for Epidemic Vaccine Trials, in: Journal of Medical Ethics 46/7 (2020) 465-469. DOI: 10.1136/medethics-2020-106235.

National Academies of Sciences, Engineering, and Medicine: Framework for Equitable Allocation of COVID-19 Vaccine. Washington, DC: National Academies Press 2020.

Nature Editors: Preparing for the Vaccine, in: Nature Biotechnology 38/11 (2020) 1219. DOI: $10.1038 / \mathrm{s} 41587-020-0743-5$.

Opolony, B.: Besuchsverbote in Pflegeeinrichtungen während der Corona-Pandemie. Infektionsschutz und Persönlichkeit, in: Zeitschrift für medizinische Ethik 67/2 (2021) 219-232. DOI: 10.14623/zfme.2021.2.219 - 232.

Persad, G./Emanuel, EJ.: The Ethics of COVID-19 Immunity-Based Licenses ("Immunity Passports”), in: Journal of the American Medical Association 323/22 (2020) 2241-2242. DOI: 10.1001/jama.2020.8102. 
Phelan, AL.: COVID-19 Immunity Passports and Vaccination Certificates: Scientific, Equitable, and Legal Challenges, in: Lancet 395/10237 (2020) 1595-1598. DOI: 10.1016/S0140-6736(20)31034-5.

Ravitsky, V./Weinstock, D.: Are Immunity Licenses Just?, in: American Journal of Bioethics 20/7 (2020) 172-174. DOI: 10.1080/15265161.2020.1779408.

Rawls, J.: Gerechtigkeit als Fairness: Ein Neuentwurf. Frankfurt am Main: Suhrkamp 2003.

Rixen, S.: Zwang in Samthandschuhen: Die Masernimpfpflicht, in: Katzenmeier, C.: Festschrift für Dieter Hart. Berlin: Springer 2020, 483-499.

Rogge, A./Naeve-Nydahl, M./Nydahl, P./Rave, F./Knochel, K./Woellert, K./ Schmalz. C.: Ethische Entscheidungsempfehlungen zu Besuchsregelungen im Krankenhaus während der COVID-19-Pandemie, in: Medizinische Klinik - Intensivmedizin und Notfallmedizin 116/5 (2021) 415-420. DOI: 10.1007/s00063021-00805-4.

Rosenbaum, L.: Escaping Catch-22 - Overcoming Covid Vaccine Hesitancy, in: New England Journal of Medicine 384/14 (2021) 1367-1371. DOI: 10.1056/NEJMms2101220.

Rosner, S.: Warum zur Eindämmung des Virus Wissen nicht reicht (2021), in: https:/www.wienerzeitung.at/nachrichten/politik/oesterreich/2094982-Warum-z ur-Eindaemmung-des-Virus-Wissen-nicht-reicht.html [09.06.2021].

Sandhofer, MJ./Robak, O./Frank, H./Kulnig, J.: Vaccine Hesitancy in Austria, in: Wiener klinische Wochenschrift 129/1 (2017) 59-64. DOI: 10.1007/s00508-0161062-1.

Schaks, N./Krahnert, S.: Die Einführung einer Impfpflicht zur Bekämpfung der Masern: Eine zulässige staatliche Handlungsoption, in: Medizinrecht 33/12 (2015) 860-866. DOI: 10.1007/s00350-015-4151-7.

Schmidt, H.: Vaccine Rationing and the Urgency of Social Justice in the Covid-19 Response, in: Hastings Center Report 50/3 (2020) 46-49. DOI: 10.1002/hast.1113.

Schröder-Bäck, P./Wild, V./Heilinger, J-C.: (2020). Vaccination Policy: Ethical Perspectives on a Future Vaccination Program against COVID-19 in Germany, in: https://www.public-health-covid19.de/images/2020/Ergebnisse/PolicyBrief_vaccination_2020_final-1.pdf [09.06.2021].

Shachar, C.: Bioethics: What Is It Good for? COVID-19 Vaccine Allocation Plans and Realities (2021), in: https://blog.petrieflom.law.harvard.edu/2021/01/26/bioethics-what-is-it-goodfor-covid-19-vaccine-allocation-plans-and-realities/ [09.06.2021].

Simon, A./Zang, C.: Herausforderungen und Chancen für die Arbeit der Akademie für Ethik in der Medizin angesichts der COVID-19-Pandemie, in: Ethik in der Medizin 32/2 (2020) 121-124. DOI: 10.1007/s00481-020-00584-0.

Trad, NK./Wharam, JF./Druss, B.: Addressing Loneliness in the Era of COVID-19, in: JAMA Health Forum 1/6 (2020) e200631. DOI: 10.1001/jamahealthforum.2020.0631. 
Usher, AD.: COVID-19 Vaccines for All?, in: Lancet 395/10240 (2020) 1822-1823. DOI: 10.1016/S0140-6736(20)31354-4.

Wallner, J.: Rechtsethik in der Medizin: Wie komme ich zu einem gut begründeten Urteil? Wien: Manz 2018.

Wiedermann-Schmidt, U.: Impfen aus Sicht der öffentlichen Gesundheit, in: Aigner, G./Grimm, M./Kletečka-Pulker, M./Wiedermann-Schmidt, U.: Schutzimpfungen - Rechtliche, ethische und medizinische Aspekte. Wien: Verlag Österreich 2016, 1-5.

Williams, JH./Dawson, A.: Prioritising Access to Pandemic Influenza Vaccine: A Review of the Ethics Literature, in: BMC Medical Ethics 21/40 (2020) 1-8. DOI: 10.1186/s12910-020-00477-3.

Wünsch-Brandner I./Grandl-Eder K.: Gesetzlich verpflichtende Impfungen für das Personal in Krankenanstalten, in: Journal für Medizin- und Gesundheitsrecht 4/2 (2019) 81-86. 
